\title{
Des prototypes rhétoriques à leur fragmentation épistolaire : l'exemple d'une consolation en pièces détachées
}

\section{Cécile Lignereux}

\section{(2) OpenEdition \\ Journals}

Édition électronique

URL : http://journals.openedition.org/rhetorique/529

DOI : 10.4000/rhetorique.529

ISSN : 2270-6909

Éditeur

UGA Éditions/Université Grenoble Alpes

Édition imprimée

ISBN : 978-2-37747-010-5

Référence électronique

Cécile Lignereux, « Des prototypes rhétoriques à leur fragmentation épistolaire : l'exemple d'une consolation en pièces détachées », Exercices de rhétorique [En ligne], 9 | 2017, mis en ligne le 04 juillet 2017, consulté le 12 septembre 2020. URL : http://journals.openedition.org/rhetorique/529; DOI : https://doi.org/10.4000/rhetorique.529

Ce document a été généré automatiquement le 12 septembre 2020.

\section{c) (i) (2)}

Les contenus de la revue Exercices de rhétorique sont mis à disposition selon les termes de la Licence Creative Commons Attribution - Pas d'Utilisation Commerciale - Partage dans les Mêmes Conditions 4.0 International. 


\title{
Des prototypes rhétoriques à leur fragmentation épistolaire : l'exemple d'une consolation en pièces détachées
}

\author{
Cécile Lignereux
}

Plutôt que de porter des jugements de valeur sur les sentiments qu'éprouve Mme de Sévigné à propos de la brève existence maladive puis du décès du petit Jean-Baptiste (enfant dont Mme de Grignan accoucha prématurément et qui mourut à seize mois), par exemple en estimant que la marquise " ne manifeste qu'une vague pitié ${ }^{1}$ » ou en jugeant qu'elle ressent au contraire "émotion et attendrissement ${ }^{2}$ ", sans doute est-il temps de substituer aux interprétations psychologiques des descriptions rhétoriques étant entendu que dans l'appréciation des témoignages de tendresse maternels, la méconnaissance des usages propres à la civilité épistolaire est à l'origine de bien des contresens. À trop vite réduire les missives à Mme de Grignan à des lettres d'amour motivées par les seuls affects d'une mère abusive, grand est le risque de négliger les représentations et les pratiques collectives dans lesquelles elles s'enracinent. Il suffit pourtant de confronter la correspondance de Mme de Sévigné aux manuels d'art épistolaire pour y repérer toute une série de rituels caractéristiques de la sociabilité de son temps - parmi lesquels la condoléance (qui vise à « témoigner la part qu'on prend à la douleur de quelqu'un ", à " participer à la douleur de quelqu'un, témoigner qu'on prend part à son déplaisir ${ }^{3}$ ») et la consolation (dont le but est de "soulager, adoucir, diminuer l'affliction, la douleur d'une personne $\left.{ }^{4} »\right)$.

2 Certes, dans les lettres du mois de juillet 1677 qui suivent la mort du jeune enfant, Mme de Sévigné ne rédige aucune lettre de consolation en bonne et due forme, se dispensant d'usages conformistes bien peu adaptés à la relation qu'elle entretient avec sa correspondante (l'intimité affective entre une mère et sa fille), au genre qu'elle pratique (la lettre familière, à raison de deux échanges par semaine) et aux valeurs stylistiques qui sont celles de son milieu (le naturel). Ni Mme de Sévigné n'exploite les 
thèmes hérités de la philosophie antique $\mathrm{e}^{5}$, ni elle ne convoque les procédés de style les plus voyants $\mathrm{du}$ discours consolatoire ${ }^{6}$, ni elle ne sollicite les raisonnements préfabriqués couramment utilisés en pareille situation ${ }^{7}$, ni elle ne mobilise l'ordonnancement canonique des différentes séquences composant le canevas standard d'une lettre de consolation ${ }^{8}$ - ce qui n'est guère étonnant compte tenu de la progressive mutation des usages consolatoires, sous l'influence à la fois de l'évolution des sensibilités (la consolation devenant « une pratique qui perd de son évidence au fur et à mesure de l'évolution des spiritualités au cours du XVII ${ }^{e}$ siècle $\left.^{9} »\right)$ et des changements de goût en matière de style (l'exigence de discrétion ayant succédé à la solennité ostentatoire des premières décennies du siècle $\left.{ }^{10}\right)$. Pourtant, bien que lapidaires et glissées parmi des rubriques épistolaires parfaitement hétérogènes (nouvelles de la santé maternelle mise à mal par une crise de rhumatisme, reproches et requêtes concernant les retrouvailles des deux femmes, anecdotes mondaines, réflexions spirituelles, badinages caractéristiques de la galanterie, inquiétudes causées par la carrière militaire de Charles de Sévigné), de nombreuses séquences éparpillées au fil des lettres relèvent de la logique rhétorique propre à la consolation - ce qui ne manque d'ailleurs pas de confirmer que, loin de fonctionner comme des protocoles aussi rigides qu'immuables, les rituels épistolaires constituent de simples moyens d'expression, éminemment personnalisables.

3 Afin d'étudier la dynamique rhétorique des séquences consolatoires dans les lettres à Mme de Grignan, nous procéderons en deux temps. D'abord, nous montrerons la cohérence d'un ensemble d'éléments textuels disséminés qui convergent au service d'une même visée persuasive - consoler une mère affligée pour qu'elle cesse de pleurer la perte de son enfant. Ensuite, nous examinerons le rendement argumentatif de séquences dont l'efficacité repose sur la sollicitation de raisonnements topiques dûment répertoriés par les manuels d'art épistolaire de l'époque.

\section{Le morcellement du discours consolatoire en contexte de familiarité épistolaire}

4 C'est dans la lettre datée du 3 juillet 1677 (commencée à Livry le 3 et continuée à Paris quelques jours plus tard) que Mme de Sévigné réagit à l'annonce du décès de son petitfils prématuré. Le dessein persuasif propre au discours consolatoire, qui consiste à tâcher de raisonner le destinataire endeuillé afin qu'il modère sa douleur, donne lieu à plusieurs séquences dispersées au fil de la lettre. Dans la première de ces séquences, qui ouvre sa missive, Mme de Sévigné met en pratique « la logique binaire et antithétique constitutive du genre de la consolation ${ }^{11}$ ", puisque d'un côté, elle exprime la part qu'elle prend au chagrin de sa destinataire (phase de condoléance) et que de l'autre, elle invoque des arguments incitant Mme de Grignan à relativiser sa perte (phase de consolation $^{12}$ ). Loin de donner lieu à une succession de deux blocs argumentatifs antinomiques qui se signalerait par son formalisme artificiel, ce "mécanisme si typique ${ }^{13} » d u$ discours consolatoire fait l'objet d'une réduplication, de telle sorte que l'on peut isoler deux sous-séquences au fonctionnement identique. La première sousséquence enchaîne rapidement la condoléance, dans laquelle Mme de Sévigné dit partager la douleur de sa fille en employant des procédés de style aussi attendus que l'isotopie de la compassion et la modalité exclamative ("Hélas! ma chère, que je suis fâchée de votre pauvre petit enfant! Il est impossible que cela ne touche»), et la 
consolation, dont l'unique argument cherche à apporter du réconfort à Mme de Grignan ("Ce n'est pas, comme vous savez, que j'ai compté sur sa vie. Je le trouvais, de la manière dont on me l'avait dépeint, sans aucune espérance »). Adoptant la même logique concessive (du type certes... mais ${ }^{14}$ ), la seconde sous-séquence exprime d'abord la sympathie de Mme de Sévigné, qui dit comprendre les raisons de l'affliction de sa fille («mais enfin c'est une perte pour vous; en voilà trois $\left.{ }^{15} »\right)$, avant de tenter d'atténuer sa douleur en l'engageant à se concentrer sur les deux enfants qui vivent auprès d'elle - le passage se terminant même par une plaisante expression familière qu'avait déjà utilisée la marquise pour se réjouir de la ressemblance entre Pauline et sa mère (« Hélas! Que je serais aise que Pauline vous ressemblât! Quelle joie de revoir ce petit chien de visage!", 15 juin 1676: II, 320). Ce qui frappe, c'est la flagrante disproportion entre d'une part, un témoignage de compassion réduit au minimum, et d'autre part, des considérations complaisamment développées sur les qualités de LouisProvence et de Pauline - une disproportion aisément compréhensible toutefois, dans la mesure où les épistoliers savent que les développements élégiaques risquent d'accroître l'affliction du destinataire endeuillé ${ }^{16}$.

Hélas! ma chère, que je suis fâchée de votre pauvre petit enfant! Il est impossible que cela ne touche. Ce n'est pas, comme vous savez, que j'ai compté sur sa vie. Je le trouvais, de la manière dont on me l'avait dépeint, sans aucune espérance, mais enfin c'est une perte pour vous ; en voilà trois. Dieu vous conserve le seul qui vous reste ! Il me paraît déjà un fort honnête homme. J'aimerais mieux son bon sens et sa droite raison que toute la vivacité de ceux qu'on admire à cet âge, et qui sont des sots à vingt ans. Soyez contente du vôtre, ma fille, et menez-le doucement, comme un cheval qui a la bouche délicate, et souvenez-vous de ce que je vous ai dit sur sa timidité. Ce conseil vient de gens plus habiles que moi, mais l'on sent qu'il est fort bon. Pour Pauline, j'ai une petite chose à vous dire. C'est que de la façon dont vous me la représentez, elle pourrait fort bien être aussi belle que vous ; voilà justement comme vous étiez. Dieu vous préserve d'une pareille ressemblance, et d'un cœur fait comme le mien! Enfin je vois qu'elle est aimable, et qu'elle vous divertit. Je voudrais bien la pouvoir embrasser, et reconnaître ce chien de visage que j'ai vu quelque part. (3 juillet $1677:$ II, 481)

5 Après avoir rapidement donné de ses nouvelles, Mme de Sévigné consacre un deuxième passage au décès du petit prématuré, passage qui, de prime abord, ne semble comprendre ni condoléance ni consolation à proprement parler. Il mérite cependant d'être cité, dans la mesure où Mme de Sévigné y dévoile explicitement les motivations qui la poussent à tenter de diminuer la peine de sa fille, à savoir encourager Mme de Grignan à ne pas affaiblir sa constitution fragile par un chagrin excessif (« Vous voulez me persuader la dureté de votre cœur pour me rassurer sur la perte de votre enfant ») :

Vous me dites que je suis en peine de votre maigreur; je vous l'avoue. C'est qu'elle parle et dit votre mauvaise santé. Votre tempérament, c'est d'être grasse, si ce n'est, comme vous dites, que Dieu vous punisse d'avoir voulu détruire une si belle santé et une machine si bien composée; c'est une si grande rage que de pareils attentats que Dieu est juste quand il les punit. Mais ceux qui en sont affligés ont, ce me semble, beaucoup de raison de l'être. Vous voulez me persuader la dureté de votre cœur pour me rassurer sur la perte de votre enfant. Je ne sais, ma fille, où vous prenez cette dureté; je ne la trouve que pour vous. Mais pour moi, et pour tout ce que vous devez aimer, vous n'êtes que trop sensible. C'est votre plus grand mal. Vous en êtes dévorée et consumée. Ah! ma chère, prenez sur nous, et donnezle au soin de votre personne. Comptez-vous pour quelque chose, et nous vous serons obligés. Vous ne sauriez rien faire pour moi qui me touche le cœur plus sensiblement. Je suis étonnée que le petit Marquis et sa sœur n'aient point été 
fâchés du petit frère. Cherchons un peu où ils auraient pris ce cœur tranquille; ce n'est pas chez vous assurément. (3 juillet 1677 : II, 482)

Ce n'est qu'après avoir évoqué l'obligation de Charles de repartir à la guerre et fait allusion aux frasques sentimentales de celui-ci que Mme de Sévigné clôt sa lettre par un " compliment ${ }^{17}$ » destiné à $\mathrm{M}$. de Grignan, qu'elle ne fait cependant que sur le mode de la prétérition ("Je devais bien faire un compliment à M. de Grignan »). Restreignant son compliment à la consolation, elle se contente d'un seul argument, présenté comme irréfutable pour tout chrétien, à savoir la félicité du nouvel ange :

Je devais bien faire un compliment à $\mathrm{M}$. de Grignan sur la mort de ce petit, mais quand on songe que c'est un ange devant Dieu, le mot de douleur et d'affliction ne peut se prononcer. Il faut que des chrétiens se réjouissent, s'ils ont le moindre principe de la religion qu'ils professent. (3 juillet 1677 : II, 482-483)

Reprise et poursuivie quatre jours plus tard, cette lettre revient une dernière fois sur la mort du prématuré à l'occasion des recommandations sur la santé que Mme de Sévigné a l'habitude de formuler en fin de lettre. L'efficacité persuasive de l'exhortation à l'impératif («Détournez-vous») est accrue par la reprise des propos que Mme de Grignan elle-même a tenus dans sa lettre précédente ("Vous dites si bien ${ }^{18}$ »), dont la modalité déontique souligne le caractère blasphématoire que revêtirait une affliction prolongée ${ }^{19}$.

Dans votre loisir, occupez-vous de votre santé. Détournez-vous de la triste pensée de la mort de cet enfant, c'est un dragon, quand on y pense trop. Vous dites si bien, il faut faire honneur au christianisme de ne pas pleurer le bonheur de ces petits anges. (7 juillet $1677:$ II, 484)

8 Les trois lettres du mois de juillet qui suivent celle-ci reviennent toutes sur le sujet de la mort du jeune Jean-Baptiste, dans le but d'aider Mme de Grignan à surmonter cette épreuve avec courage et fermeté. Dans la lettre du 9 juillet 1677, c'est le thème de la santé qui donne lieu à une exhortation caractéristique de la rhétorique consolatoire ("Vous ne devriez penser [...] qu'à vous détourner, tant que vous pourriez, de la pensée de ce pauvre petit garçon que vous avez perdu »). Les mauvaises nouvelles concernant l'état de santé de Mme de La Fayette n'ayant pas manqué de raviver les inquiétudes maternelles à l'égard d'une fille prématurément affaiblie par diverses pathologies pulmonaires et circulatoires, l'appel au contrôle de soi, loin de manifester une quelconque option spirituelle ou philosophique, relève du bon sens : si Mme de Grignan ne doit pas se contenter de faire illusion en tenant de «beaux discours» rationnels, c'est que le «dragon » (expression souvent utilisée par Mme de Sévigné pour désigner un sujet d'inquiétude) que constitue la pensée de son enfant décédé risque fort d'affaiblir sa santé déjà chancelante :

Cela m'attriste, et pour elle que j'aime fort et pour ceux qui ont le sang si extrêmement subtil; il me semble qu'il ne faut rien pour embraser toute la machine. Ma fille, quand on aime bien, il n'est pas ridicule de souhaiter qu'un sang auquel on prend tant d'intérêt se tranquillise et se rafraîchisse. Vous ne devriez penser, ce me semble, qu'à épaissir le vôtre, et qu'à vous détourner, tant que vous pourriez, de la pensée de ce pauvre petit garçon que vous avez perdu. J'ai peur qu'avec tous vos beaux discours, vous ne vous en fassiez un dragon. Ma très chère, ayez pitié de vous et de moi. (9 juillet 1677 : II, 485)

9 La séquence consolatoire de la lettre datée du 14 juillet 1677 est déclenchée par la venue à Grignan du médecin Amonio, Mme de Sévigné regrettant que le petit prématuré n'ait pas pu recevoir des soins, qui, selon elle, auraient été plus adaptés. Immédiatement après avoir formulé cet irréel du passé, elle s'efforce toutefois d'ôter 
tout regret à Mme de Grignan en invoquant les desseins de la Providence et en rappelant le rachitisme de l'enfant, qui de toute évidence ne pouvait pas survivre (« mais je ne croyais pas qu'il dût vivre avec de tels accidents ») - même si durant sa brève existence, Mme de Sévigné n'avait pas manqué, quoi qu'elle en dise une fois l'enfant décédé, d'avoir quelques lueurs d'espoir. Consciente qu'une consolation aussi brutale risque de heurter une destinataire encore très affectée par la perte de son enfant, Mme de Sévigné exprime aussitôt sa condoléance, clairement signalée par des marqueurs lexicaux aussi courants que les verbes comprendre et sentir («je la comprends et je la sens comme elle est ») - ce qui confirme bien le caractère périlleux de l'art de consoler, qui peut à tout moment être perçu comme une intrusion, voire une agression :

\begin{abstract}
Je suis bien aise que vous soyez contente d'Amonio ; si vous l'aviez eu, sans doute il aurait sauvé votre fils. Il fallait le rafraîchir ; l'ignorance me paraît grande de l'avoir échauffé, mais la difficulté était de déranger tout ce qu'avait réglé la Providence en faveur de ce pauvre enfant. Cette affliction est du nombre de celles où l'on doit se soumettre sans murmurer à ce qu'elle ordonne. Il est vrai que je n'avais point du tout compté sur sa vie. Où avez-vous pris, ma bonne, qu'un enfant qui n'a point de dents, et qui ne se soutient pas à dix-huit mois, ait échappé tous les périls? Je ne suis pas si éclairée que Mme du Puy-du-Fou, mais je ne croyais pas qu'il dût vivre avec de tels accidents. C'est ce qui m'a ôté toute surprise pour la perte de ce troisième garçon ; je la comprends et je la sens comme elle est. Pauline me ravit. J'ai parlé tantôt au bel abbé d'un précepteur que connaît M. de La Mousse; ils se verront et vous en diront leur avis. Ils disent que le Marquis est bien jeune; j'ai répondu que son esprit ne l'était pas. (14 juillet 1677 : II, 486)
\end{abstract}

La lettre du 16 juillet est la dernière de la série à revenir sur le décès du prématuré. L'ultime séquence consolatoire intervient en fin de lettre, au moment des adieux, à la suite de toutes sortes de rubriques (rythmes épistolaires, anecdotes mondaines, affaires, réflexions théologiques, copie d'un portrait de Mme de Grignan, conseils sur l'éducation de Louis-Provence, départ imminent de Charles de Sévigné, qui ne peut plus se soustraire à ses obligations militaires). La consolation prend la forme d'une exhortation («Consolez-vous du petit») suivie de raisonnements médicaux visant à ôter à Mme de Grignan tout sentiment de culpabilité quant aux soins qui auraient pu sauver son enfant. Si cette séquence consolatoire se clôt de manière relativement abrupte, c'est que Mme de Sévigné a bien l'intention de signifier à sa fille qu'il est désormais temps de dépasser son chagrin : en faisant suivre le raisonnement médical, formulé à la manière d'une vérité générale, d’une chute qui confine au burlesque ( Je parle d'or »), l'épistolière donne explicitement un coup d'arrêt à la dynamique consolatoire - et de fait, à partir de la lettre suivante, il ne sera plus question ni du prématuré ni du chagrin de Mme de Grignan.

Adieu, ma très chère. Consolez-vous du petit ; il n'y a de la faute de personne. Il est mort des dents, et non pas d'une fluxion sur la poitrine. Quand les enfants n'ont pas la force de les pousser dans le temps, ils n'ont pas celle de soutenir le mouvement qui les veut faire percer toutes à la fois. Je parle d'or. (16 juillet 1677 : II, 493)

11 Ainsi donc, malgré leurs différences (en termes d'étendue textuelle, de mode d'intégration au sein de la lettre, d'équilibre syntagmatique entre condoléance et consolation, de tonalité), les séquences mises en série s'avèrent toutes motivées par la visée persuasive propre au discours consolatoire, puisque, chacune à leur manière, elles visent à aider Mme de Grignan à surmonter son chagrin. Incontestablement, au moment où Mme de Sévigné tente d'apporter du réconfort à sa fille endeuillée, "c'est Françoise qu'elle plaint sans cesse et pour la santé de laquelle elle s'inquiète beaucoup ${ }^{20}$ 
». Cependant, on aurait tort de voir dans cette attitude la preuve d'un amour maternel anormalement violent, dans la mesure où le discours consolatoire de Mme de Sévigné mobilise des arguments aussi ordinaires que codifiés - ce qui confirme, s'il en était encore besoin, que dans les correspondances du Grand Siècle, les mouvements de sensibilité ne sauraient être communiqués en dehors de rouages discursifs scrupuleusement normalisés.

\section{2. Émiettement et ventilation des arguments consolatoires}

À l'opposé aussi bien d'une soumission conformiste à des règles de bienséance sclérosées que d'une impensable singularisation de soi pensée en termes d'inventivité langagière (deux écueils incompatibles avec l'exigence de naturel), les séquences consolatoires qui s'égrènent dans les lettres à Mme de Grignan des 3, 9, 14 et 16 juillet 1677 reposent sur des routines argumentatives couramment mises en œuvre dans les lettres de consolation de l'époque. À la lumière des descriptifs rhétoriques fournis par les manuels d'art épistolaire, il est possible d'en repérer quatre ${ }^{21}$.

\section{1. «Considérer le mal qu'elle a reçu22 »}

13 Dans les lettres qui suivent le décès du petit prématuré, Mme de Sévigné invoque à plusieurs reprises les desseins de la Providence. La première fois, c'est dans le compliment par prétérition qu'elle adresse à son gendre :

Je devais bien faire un compliment à $\mathrm{M}$. de Grignan sur la mort de ce petit, mais quand on songe que c'est un ange devant Dieu, le mot de douleur et d'affliction ne peut se prononcer. Il faut que des chrétiens se réjouissent, s'ils ont le moindre principe de la religion qu'ils professent. (3 juillet 1677 : II, 482-483)

La deuxième fois, c'est pour encourager Mme de Grignan à se ménager, ce qui implique de ne pas prolonger un état d'affliction dommageable à sa santé :

Dans votre loisir, occupez-vous de votre santé. Détournez-vous de la triste pensée de la mort de cet enfant, c'est un dragon, quand on y pense trop. Vous dites si bien, il faut faire honneur au christianisme de ne pas pleurer le bonheur de ces petits anges. (7 juillet $1677:$ II, 484)

La troisième fois, c'est pour empêcher Mme de Grignan de se perdre en conjectures sur les causes de la mort de son enfant et sur les soins qui auraient pu le sauver :

Je suis bien aise que vous soyez contente d'Amonio; si vous l'aviez eu, sans doute il aurait sauvé votre fils. Il fallait le rafraîchir ; l'ignorance me paraît grande de l'avoir échauffé, mais la difficulté était de déranger tout ce qu'avait réglé la Providence en faveur de ce pauvre enfant. Cette affliction est du nombre de celles où l'on doit se soumettre sans murmurer à ce qu'elle ordonne. (14 juillet 1677 : II, 486)

Certes, il peut être tentant d'expliquer l'attitude dogmatique de l'épistolière ( Il faut que des chrétiens se réjouissent », « il faut faire honneur au christianisme », « l'on doit se soumettre sans murmurer ») par ses options spirituelles - un christianisme fervent, des lectures jansénistes, un attachement croissant à la théologie de la Providence. Pourtant, ce serait faire fausse route que de rapporter ces arguments consolatoires à de simples particularités biographiques. L'argument qui consiste à se soumettre à la Providence avec résignation afin de retrouver la paix et la sérénité constitue en effet l'un des arguments topiques de la lettre de consolation, systématiquement mentionné dans les descriptifs rhétoriques des manuels d'art épistolaire ${ }^{23}$. Conseiller de s'en 
remettre à la Providence "sans murmurer" (expression caractéristique de la phraséologie consolatoire) pour trouver l'apaisement dans l'épreuve est d'autant plus naturel et facile à l'épistolière que les parents affligés eux-mêmes ne manquent pas d'alléguer la volonté divine, comme le prouvent les deux passages au discours rapporté. C'est le cas de Mme de Grignan, dont Mme de Sévigné approuve les propos dans sa lettre du 7 juillet (« Vous dites si bien, il faut faire honneur au christianisme de ne pas pleurer le bonheur de ces petits anges »). C'est aussi le cas de M. de Grignan, qui, dès la naissance de Jean-Baptiste, fait des "réflexions chrétiennes", devinant qu'à une époque où la médecine s'avère impuissante à sauver les enfants nés avant terme et de mauvaise conformation, son enfant est condamné. Après avoir enchainé, dans la lettre qu'elle adresse à sa fille après l'accouchement, un compliment de conjouissance ("Quel bonheur que vous vous portiez bien!») et un compliment de condoléance (« mais quel dommage d'avoir perdu encore un pauvre petit garçon!»), Mme de Sévigné s'adresse ainsi à son gendre, qui a pris soin de lui écrire tandis que Mme de Grignan est encore alitée :

Je vous rends grâces de vos trois lignes, et à vous, mon cher Comte, des soins que vous prenez de m'instruire. Vous savez ce que c'est pour moi que la santé de votre chère femme. Mais vous l'avez laissée trop écrire ; c'est une mort que cet excès, et pour ce lavage des pieds, on dit qu'il a causé l'accouchement. C'est dommage de la perte de cet enfant ; je la sens, et j'ai besoin de vos réflexions chrétiennes pour m'en consoler, car quoi qu'on vous dise, vous ne le sauverez pas à huit mois. (23 février 1676 : II, 241)

Irréductibles à de simples leçons de pragmatisme, à de purs réflexes psychologiques ou à de fermes positions théologiques, les séquences consolatoires invoquant la soumission à la Providence reflètent surtout les usages rhétoriques du temps, l'argument de la volonté divine étant considéré comme l'un des plus efficaces pour apporter du réconfort à une personne endeuillée - et notamment à des parents (en particulier les mères, réputées plus pieuses) qui viennent de perdre un enfant ${ }^{24}$.

S'il paraît légitime à Mme de Sévigné de conseiller à Mme de Grignan de cesser de pleurer son enfant, ce n'est pas seulement parce que telle est la volonté divine. C'est aussi parce que le défunt est désormais heureux ${ }^{25}$. L'argument du bonheur des enfants morts en bas âge ${ }^{26}$, anges auprès de Dieu ${ }^{27}$, avait d'ailleurs déjà été utilisé par Mme de Sévigné, quelques mois après la naissance du petit prématuré. Prenant part à la douleur de sa fille face à l'état végétatif de son enfant, la marquise, "trop stricte catholique pour proférer un blasphème, mais trop pitoyable femme pour ne pas exprimer les vœux du bon sens ${ }^{28} "$, avait exprimé sa condoléance en ces termes :

Vous m'avez fait venir les larmes aux yeux en me parlant de votre petit. Hélas, le pauvre enfant ! le moyen de le regarder en cet état! Je ne me dédis point de ce que j'en ai toujours pensé, mais je crois que, par tendresse, on devrait souhaiter qu'il fût là où son bonheur l'appelle. (30 juin $1677:$ II, 478)

Certes, en rappelant à sa fille qu' «il faut faire honneur au christianisme de ne pas pleurer le bonheur de ces petits anges » (7 juillet 1677), Mme de Sévigné sollicite un argument tout à fait courant dans les lettres destinées à consoler des parents meurtris. Pourtant, elle le fait avec une rapidité et une discrétion qui contrastent avec le didactisme sermonnaire et la véhémence parfois railleuse de certaines lettres de consolation adressées à des mères dont l'affliction est jugée indécente ${ }^{29}$.

En exploitant à plusieurs reprises, fût-ce de manière concise et familière, les thèmes éprouvés de la volonté divine et du bonheur de l'enfant devenu ange au ciel, Mme de Sévigné illustre bien l'habitude qu'ont les épistoliers du Grand Siècle non seulement de 
solliciter les arguments canoniques qui sont à leur disposition, mais encore de les doser en fonction de la rentabilité argumentative que leur confère telle ou telle situation d'énonciation - les arguments théologiques auxquels adhère tout le milieu de la marquise figurant parmi ceux qui promettent d'être les plus efficaces auprès d'une mère endeuillée forcée d'admettre que la mort d'un enfant "n'est pas un mal ${ }^{30}$ ", puisque celui-ci est heureux auprès de Dieu.

\section{2. «Considérer la personne que regrette celle que l'on console ${ }^{31}$ »}

17 Afin d'aider Mme de Grignan à surmonter le chagrin causé par la mort du petit JeanBaptiste, Mme de Sévigné ne manque pas de rappeler à sa fille les vices de conformation qui empêchaient le prématuré d'être viable. Inciter sa fille à considérer les troubles de son enfant sous un nouvel angle, c'est-à-dire sans en nier l'extrême gravité, afin qu'elle puisse relativiser sa perte : tel est le but des passages dans lesquels la marquise souligne que l'état de l'enfant ne laissait " aucune espérance ",

Ce n'est pas, comme vous savez, que j'ai compté sur sa vie. Je le trouvais, de la manière dont on me l'avait dépeint, sans aucune espérance, mais enfin c'est une perte pour vous ; en voilà trois. ( 3 juillet $1677:$ II, 481)

qu'il ne pouvait pas survivre avec de telles anomalies de croissance (absence de dents, incapacité à marcher),

Il est vrai que je n'avais point du tout compté sur sa vie. Où avez-vous pris, ma bonne, qu'un enfant qui n'a point de dents, et qui ne se soutient pas à dix-huit mois, ait échappé tous les périls ? Je ne suis pas si éclairée que Mme du Puy-du-Fou, mais je ne croyais pas qu'il dût vivre avec de tels accidents. C'est ce qui m'a ôté toute surprise pour la perte de ce troisième garçon; je la comprends et je la sens comme elle est. (14 juillet 1677 : II, 486)

et qu'il était impensable qu'il puisse échapper au sort promis aux enfants dont les dents ne sortent pas à temps :

Adieu, ma très chère. Consolez-vous du petit ; il n'y a de la faute de personne. Il est mort des dents, et non pas d'une fluxion sur la poitrine. Quand les enfants n'ont pas la force de les pousser dans le temps, ils n'ont pas celle de soutenir le mouvement qui les veut faire percer toutes à la fois. Je parle d'or. (16 juillet 1677 : II, 493)

En insistant ainsi à trois reprises sur le fait qu'il était impossible que le petit JeanBaptiste ait pu continuer à vivre, Mme de Sévigné met en pratique l'une des « thérapies consolatoires» tout à fait usuelles - celle qui consiste à «minimiser l'étendue de la perte $^{32}$ » afin que le destinataire donne de plus justes proportions à sa peine. Si Mme de Sévigné allègue le fait que les graves troubles de croissance du prématuré ne lui laissaient aucun espoir, c'est pour suggérer le caractère déraisonnable voire absurde d'un chagrin qui n'a pas lieu de se prolonger. Le fait de relativiser un deuil au moyen de considérations sur l'état, l'âge ou le statut familial de la personne décédée fait d'ailleurs partie des réflexes que doit avoir tout épistolier ayant à rédiger une lettre de consolation, à l'instar des auteurs de manuels d'art épistolaire, qui distinguent les protocoles rhétoriques en fonction de la gravité de la perte ${ }^{33}$ et qui classent les modèles de lettre selon le degré d'affliction du destinataire ${ }^{34}$. De toute évidence, une affliction intense et prolongée est plus légitime lorsqu'il s'agit d'« un mari qui perd une femme, pour laquelle il aurait eu une grande tendresse; une jeune femme dans le même état chargée d'enfants, qui aurait perdu un époux qu'elle aurait aimé beaucoup, un père ou une mère qui ont perdu un enfant unique, en qui ils avaient mis toutes leurs espérances; ou pour la perte d'un père, ou d'une mère, ou de quelques grands amis " 
que lorsqu'il «ne s'agit que de consoler sur des maladies, des pertes d'autres enfants que d'uniques, de frère, de sœur, d'oncle et de tante, ou de quelque autre parent que ce soit, ou d'amis à l'ordinaire, de biens, de procès, qui ne touchent pas si sensiblement ${ }^{35}$ ॥.

La brusquerie dont fait preuve Mme de Sévigné dans les passages où elle évoque l'état désespéré de l'enfant peut surprendre - qu'il s'agisse de l'agressivité railleuse de l'interrogation (« Où avez-vous pris, ma bonne, qu'un enfant qui n'a point de dents, et qui ne se soutient pas à dix-huit mois, ait échappé tous les périls?») ou de l'autodérision burlesque du commentaire métadiscursif ( Je parle d'or »). En réalité, si Mme de Sévigné se moque des espérances absurdes de sa fille, c'est, en la forçant à être plus objective sur l'état du prématuré, pour l'aider à mettre des bornes à son chagrin. Autrement dit, si Mme de Sévigné ironise sur les erreurs d'appréciation de Mme de Grignan, ce n'est pas par insensibilité, mais par souci de persuader la comtesse (qui était d'ailleurs la première à reconnaître que son enfant vivait « contre toutes les règles de la médecine $\left.{ }^{36} »\right)$ de modérer sa douleur. Il suffit de se rappeler que Mme de Sévigné n'a pas manqué, au cours de la brève existence de Jean-Baptiste, de partager les regains d'optimisme de sa fille, au point même de plaisanter sur le prodige que constituait la survie de l'enfant ${ }^{37}$, pour comprendre que son insistance sur le caractère inéluctable de la mort du prématuré relève d'une tactique persuasive. Bien que l'on puisse être tenté d'interpréter les traits d'humour maternels comme des preuves du fait que Mme de Sévigné "aime ses petits-enfants moins, et autrement, que nous n'attendons d'une grand-mère ${ }^{38}$ » ou que " plus encore que l'éloignement ou les habitudes d'éducation du temps, la force de son amour pour sa fille l'a empêchée d'exercer cet art, d'être ce qu'on appelle une bonne grand-mère ${ }^{39} "$, il suffit de les confronter aux consignes et aux exemples des manuels d'art épistolaire pour constater que l'épistolière fait finalement preuve d'aménité assez inattendue. Alors que son statut maternel l'autoriserait à formuler de sévères objurgations ${ }^{40}$ à l'égard d'une fille dont le chagrin n'est pas raisonnable compte tenu des lourdes pathologies dont était affecté l'enfant décédé, Mme de Sévigné se contente d' "entremêler quelque raillerie parmi son discours" ${ }^{41}$ " pour aider Mme de Grignan à faire preuve de davantage de sagesse. Comparée à la véhémence de certains consolateurs qui n'hésitent ni à tourner en ridicule l'« excès " de chagrin et les "faiblesses" de leur destinataire ni à les accabler d'austères réprimandes en raison de l'« opiniâtreté » de leur affliction ${ }^{42}$, la manière tantôt réaliste tantôt plaisante dont Mme de Sévigné tâche de consoler sa fille en évoquant l'état de l'enfant apparait en fin de compte relativement indulgente et débonnaire. Soulignons d'ailleurs que l'humeur badine dont fait preuve Mme de Sévigné au sein même des séquences consolatoires n'a rien d'inconvenant: le décès du petit Jean-Baptiste (un enfant dont rien ne permettait ni d'espérer ni même de souhaiter la survie) ne constituant qu'une "légère infortune", l'épistolière est parfaitement autorisée à « dérider » sa destinataire « avec une plaisanterie ${ }^{43}$ ».

\section{3. «Considérer les autres personnes qui touchent celle dont on veut diminuer la douleur ${ }^{44}$ "}

20 L'argument consolatoire consiste ici à représenter au destinataire endeuillé les dégâts que son affliction pourrait causer sur les personnes de son entourage - qu'il s'agisse d'un père âgée ${ }^{45}$, d'enfants qui requièrent de l'attention ${ }^{46}$, d'un conjoint courageux, d'un frère illustre ${ }^{47}$, ou encore d'une mère inquiète - comme Mme de Sévigné. À trois reprises, celle-ci exhorte Mme de Grignan à mettre un terme à son chagrin afin de ne 
pas compromettre un état de santé déjà précaire, qui ne manque pas de susciter chez elle les plus vives inquiétudes ${ }^{48}$ - qu'il s'agisse de la maigreur de la comtesse,

Vous me dites que je suis en peine de votre maigreur; je vous l'avoue. C'est qu'elle parle et dit votre mauvaise santé. Votre tempérament, c'est d'être grasse, si ce n'est, comme vous dites, que Dieu vous punisse d'avoir voulu détruire une si belle santé et une machine si bien composée; c'est une si grande rage que de pareils attentats que Dieu est juste quand il les punit. Mais ceux qui en sont affligés ont, ce me semble, beaucoup de raison de l'être. Vous voulez me persuader la dureté de votre cœur pour me rassurer sur la perte de votre enfant. (3 juillet 1677 : II, 482)

de son organisme épuisé qui nécessite des soins et du repos,

Dans votre loisir, occupez-vous de votre santé. Détournez-vous de la triste pensée de la mort de cet enfant, c'est un dragon, quand on y pense trop. (7 juillet 1677 : II, 484)

ou d'un sang que la médecine de l'époque considère comme trop subtil et trop échauffé :

Cela m'attriste, et pour elle que j'aime fort et pour ceux qui ont le sang si extrêmement subtil; il me semble qu'il ne faut rien pour embraser toute la machine. Ma fille, quand on aime bien, il n'est pas ridicule de souhaiter qu'un sang auquel on prend tant d'intérêt se tranquillise et se rafraîchisse. Vous ne devriez penser, ce me semble, qu'à épaissir le vôtre, et qu'à vous détourner, tant que vous pourriez, de la pensée de ce pauvre petit garçon que vous avez perdu. J'ai peur qu'avec tous vos beaux discours, vous ne vous en fassiez un dragon. Ma très chère, ayez pitié de vous et de moi. (9 juillet 1677 : II, 485)

21 À chaque fois, ce sont des recommandations sur la santé qui débouchent sur des objurgations consolatoires, explicitées dans les deux dernières séquences au moyen du verbe se détourner («détournez-vous» et «vous ne devriez penser [...] qu'à vous détourner »). Le mouvement qui conduit du général (une santé délicate qu'il convient de ménager) au particulier (la tristesse résultant du décès du petit prématuré, qui constitue une nouvelle menace pour cette santé) ne manque pas de renforcer l'impact persuasif des objurgations qui visent à faire prendre conscience à Mme de Grignan de la nécessité de mettre un terme à une affliction qui ne peut qu'accentuer les symptômes dont elle souffre. Si Mme de Sévigné semble accorder davantage d'importance à l'état physique de sa fille qu'à ses sentiments, c'est qu'avec un bon sens tout maternel, elle devine qu'une nouvelle détérioration de sa santé sera irrémédiable, alors que son chagrin s'atténuera nécessairement au fil du temps. En avertissant Mme de Grignan des maux que lui fait courir un chagrin excessif, Mme de Sévigné ne se montre ni scandaleusement indiscrète ni particulièrement autoritaire, comme certains critiques mal informés des usages de l'époque ont pu le penser : elle s'acquitte de l'un des devoirs des parents vigilants et attentionnés, qui consiste à mettre en garde leurs enfants contre les dangers (qu'ils soient d'ordre physique, spirituel ou social) que fait courir une affliction immodérée - les avertissements, exhortations et objurgations faisant partie intégrante des protocoles persuasifs de la lettre de consolation, lorsque celle-ci est écrite par un épistolier qui bénéficie d'une quelconque supériorité hiérarchique sur son destinataire.

Certes, il est tentant de qualifier d'égoïstes et de tyranniques les objurgations maternelles qui tentent de persuader Mme de Grignan de prendre soin de sa santé en cessant de s'affliger, dans le but d'épargner à sa mère des inquiétudes et des angoisses supplémentaires :

Comptez-vous pour quelque chose, et nous vous serons obligés. Vous ne sauriez rien faire pour moi qui me touche le cœur plus sensiblement. (3 juillet 1677 : II, 482) 
J'ai peur qu'avec tous vos beaux discours, vous ne vous en fassiez un dragon. Ma très

chère, ayez pitié de vous et de moi. (9 juillet 1677 : II, 485)

Pourtant, ne nous y trompons pas : loin de résulter d'un amour exceptionnellement possessif qui utiliserait les conseils en matière de santé comme autant de moyens de pression et de chantage, les supplications qui font dépendre le bien-être maternel de la santé de Mme de Grignan ne font que mettre en œuvre l'argument consolatoire qui consiste à « considérer les autres personnes qui touchent celle dont on veut diminuer la douleur ».

\section{4. « Diminuer le regret des choses passées, par l'espérance des futures ${ }^{49} »$}

À première vue, le choix d'intégrer à l'intérieur des séquences de consolation les diverses considérations sur les deux enfants qui vivent auprès de Mme de Grignan (Marie-Blanche ayant pour sa part été placée chez les Visitandines dès l'âge de cinq ans et demi) peut surprendre. Indéniablement, on pourrait être tenté d'exclure du discours consolatoire ces propos empreints de tendresse, de confiance et d'espièglerie - c'est-àdire de diagnostiquer un simple changement de sujet, dont la promptitude est d'autant plus opportune qu'elle évite d'évoquer trop longuement «la perte que l'on a faite ${ }^{50}$ ». Toutefois, ce serait méconnaître l'un des principaux arguments des lettres de consolation, qui consiste à "consoler par l'espérance d'une meilleure fortune ${ }^{51}$ ». Certes, Mme de Sévigné épargne à sa fille les réflexions aussi explicites que pesantes sur le fait que d'autres enfants lui sont «laissés» par «la Providence» pour la "dédommager ${ }^{52}$ ", que ceux-ci sont "plus agréables ${ }^{53}$ " que celui qu'elle vient de perdre, qu'ils sont «de grande espérance ${ }^{54} »$ ou qu'ils lui «donneront toujours de la satisfaction $^{55}$ ». Néanmoins, en choisissant de clore les principales séquences de consolation sur le thème des progrès faits par les deux enfants, Mme de Sévigné n'en actualise pas moins l'un des maillons argumentatifs du discours consolatoire - celui qui consiste à mettre l'accent sur les sources de contentement sur lesquelles le destinataire peut compter ${ }^{56}$. Tantôt elle loue un petit-fils qui, en dépit d'une grande timidité, lui semble "déjà un fort honnête homme ", et une petite-fille qu'elle juge "belle » et « aimable» :

Dieu vous conserve le seul qui vous reste! Il me paraît déjà un fort honnête homme. J'aimerais mieux son bon sens et sa droite raison que toute la vivacité de ceux qu'on admire à cet âge, et qui sont des sots à vingt ans. Soyez contente du vôtre, ma fille, et menez-le doucement, comme un cheval qui a la bouche délicate, et souvenezvous de ce que je vous ai dit sur sa timidité. Ce conseil vient de gens plus habiles que moi, mais l'on sent qu'il est fort bon. Pour Pauline, j'ai une petite chose à vous dire. C'est que de la façon dont vous me la représentez, elle pourrait fort bien être aussi belle que vous; voilà justement comme vous étiez. Dieu vous préserve d'une pareille ressemblance, et d'un cœur fait comme le mien! Enfin je vois qu'elle est aimable, et qu'elle vous divertit. Je voudrais bien la pouvoir embrasser, et reconnaître ce chien de visage que j'ai vu quelque part. (3 juillet 1677 : II, 481)

Tantôt elle se réjouit de l'égalité d'humeur d'enfants qui n'ont visiblement pas été affectés par la maladie puis le décès de leur petit frère prématuré :

Je suis étonnée que le petit Marquis et sa sœur n'aient point été fâchés du petit frère. Cherchons un peu où ils auraient pris ce cœur tranquille; ce n'est pas chez vous assurément. (3 juillet 1677 : II, 482)

Tantôt elle souligne la vivacité de Pauline et la précocité intellectuelle de LouisProvence : 
Pauline me ravit. J'ai parlé tantôt au bel abbé d'un précepteur que connaît M. de La Mousse ; ils se verront et vous en diront leur avis. Ils disent que le Marquis est bien jeune ; j'ai répondu que son esprit ne l'était pas. (14 juillet 1677 : II, 486) Mme de Grignan des 3, 9, 14 et 16 juillet 1677, il est désormais possible d'en caractériser le fonctionnement rhétorique - ce qui permet au passage de récuser un certain nombre d'idées reçues sur la démarche consolatoire en contexte familier qui, pour être séduisantes, n'en sont pas moins fausses.

La première méprise concerne l'inventio. Si Mme de Sévigné se dispense aisément de reproduire l'intégralité des canevas préconisés par les manuels, et donc d'actualiser toutes les topiques consolatoires disponibles en même temps ${ }^{59}$, elle n'en utilise pas moins les arguments canoniques qui lui paraissent les plus susceptibles d'aider sa fille à surmonter sa douleur (une affliction qu'il n'est pas légitime de prolonger, une personne qu'il n'est pas raisonnable de regretter, un entourage qu'il faut préserver, des espérances auxquelles il faut accorder la priorité). Il convient donc de résister aux fantasmes caricaturaux sur le style épistolaire à l'âge classique, aussi bien à celui du formalisme stéréotypé qui serait censé régir les correspondances qu’à celui de la créativité débridée que parviendraient à conquérir les épistoliers les plus talentueux manière de réaffirmer la nécessité de raisonner en termes de sélection, de dosage et de redistribution.

La deuxième illusion d'optique se rapporte à la dispositio. Alors que l'on pourrait ne voir dans les séquences consolatoires disséminées ici et là qu'une suite hasardeuse de réactions intempestives de la part d'une mère passionnée soucieuse d'atténuer le chagrin de sa fille, il convient d'en remarquer non seulement la cohérence mais encore la progression argumentatives. Plutôt que de croire que ces unités textuelles disjointes ne convergent pas en un argumentaire et que l'ordre des arguments avancés ne répond à aucun schéma d'ensemble (comme si seul un discours monolithique pouvait être modelé par une visée argumentative organisatrice), il convient d'en mettre au jour la solidarité et la congruence au service d'une tension persuasive. Dès lors que l'on envisage les séquences consolatoires, aussi dispersées soient-elles, non pas comme une succession aléatoire de fragments autonomes, mais comme une organisation 
argumentative discontinue, se manifestent trois sortes de réglages dispositionnels: d'une part, l'évacuation de la condoléance au profit de la seule consolation (déjà réduite à la portion congrue dans la première séquence, la condoléance disparaît totalement des suivantes, à l'exception du bref compliment glissé dans la lettre du 14 juillet 1677); d'autre part, le choix systématique de clore les plus longues séquences consolatoires par des considérations sur les enfants qui vivent auprès de Mme de Grignan; enfin, l'infléchissement du discours consolatoire dans le sens d'une sévérité accrue à l'égard de l'état d'esprit de la comtesse, la progressive prépondérance des objurgations étant marquée par la montée en puissance de la raillerie. Ce n'est donc pas parce que le discours consolatoire se trouve morcelé au fil de différentes lettres qu'il ne procède pas selon une trajectoire argumentative.

La troisième méprise se situe sur le plan de l'elocutio. À force que les lettres de consolation de l'âge baroque (morceaux de bravoure constamment réimprimés au XVII siècle, pour le plus grand plaisir des lecteurs ${ }^{60}$ ) aient tendance à monopoliser l'attention, grand est le risque de croire que le discours consolatoire soit voué à une éloquence solennelle, érudite et copieuse. Certes, la consolation, telle qu'elle est décrite et illustrée par les manuels d'art épistolaire, fait volontiers usage d'ornements stylistiques spectaculaires, parmi lesquels «l'Interrogation, l'Exclamation, l'Admiration, la Prière et l'Apostrophe ", "afin que l'esprit des affligés en soit touché plus vivement ${ }^{61} »$. Pourtant, dans le cadre d'un échange épistolaire familier et à une époque où se multiplient les réticences à l'égard d'un rituel socio-discursif passablement discrédité ${ }^{62}$, l'entreprise consolatoire paraît surtout régie par un impératif de brièveté - une brièveté qui en conditionne l'efficacité, à l'opposé des " grandes lettres de consolation qui n'ont jamais nul effet ${ }^{63}$ » dont se moque Mlle de Scudéry. En choisissant de ne formuler que des consolations «succinctes, quoique sur le ton plaintif ${ }^{64}$ ", Mme de Sévigné trouve le moyen de faire preuve de compassion « sans aller faire de longues plaintes » ni « de longues exagérations contre la cruauté de la mort ${ }^{65} »$; d'exhorter sa destinataire à modérer son affliction sans avoir l'air de dispenser quelque «commandement ${ }^{66}$ » autoritaire; de railler plaisamment le caractère déraisonnable du chagrin de Mme de Grignan sans se complaire dans d'ingénieux raisonnements qui pourraient « avoir quelque chose de brillant ou de trop recherché67 $^{\prime}$; d'aider sa fille à relativiser sa perte par des considérations chrétiennes sans tomber dans les envolées lyriques propres aux " oraisons funèbres ${ }^{68}$ » - bref, de n'employer que "des expressions naturelles et tendres ${ }^{69}$ ». À cet égard, le choix de fragmenter le discours consolatoire en de courtes séquences parfaitement intégrées au reste du dialogue épistolaire constitue une authentique stratégie rhétorique, fondée sur la volonté de n'écrire que des consolations «raisonnables", c'est-à-dire "sans employer toute la Morale, et toute l'éloquence inutilement ${ }^{70} »$.

\section{NOTES}

1. J. Duchêne, " "Mes petites entrailles", ou l'art d'être grand-mère de Mme de Sévigné ", dans R. Duchêne et P. Ronzeaud dir., Autour de Mme de Sévigné. Deux colloques pour un Tricentenaire: 
Rapports mère-fille au XVII e siècle et de nos jours (Marseille, 20-21 janvier 1996); Les voyages en France au XVII siècle (Aix-en-Provence, 27-28 octobre 1996), Paris-Seattle-Tübingen, Papers on French Seventeenth Century Literature, « Biblio $17 », 105,1997$, p. 56.

2. Th. Lassalle, «Une grand-mère au XVII ${ }^{\mathrm{e}}$ siècle, Mme de Sévigné ", Littératures classiques, $\mathrm{n}^{\circ} 14$, janvier 1991, p. 176.

3. Dictionnaire de l'Académie française, Paris, Veuve Jean-Baptiste Coignard, 1694, s.v. condoléance et condouloir - nous modernisons l'orthographe de toutes nos sources. Sur les préconstruits sociodiscursifs et sur les marqueurs stylistiques de l'expression de la condoléance dans les lettres de Mme de Sévigné à Mme de Grignan, voir C. Lignereux, «Une routine de la civilité épistolaire : l'expression de la condoléance ", Exercices de rhétorique [En ligne], 6|2016, URL: http:// rhetorique.revues.org/437.

4. Dictionnaire de l'Académie française, s.v. consoler. Il s'avère indispensable de distinguer les deux démarches rhétoriques, même si la condoléance et la consolation « ont tendance à s'interpénétrer » et que « les manuels hésitent beaucoup dans leur terminologie, ne faisant pas de nette distinction entre les deux genres", comme le souligne Th. Carr, "Se condouloir ou consoler? Les condoléances dans les manuels épistolaires de l'ancien régime", Studies on Voltaire and the eighteenth century, 358, Voltaire Foundation, Oxford, 1997, p. 220-221. Th. Carr résume ainsi les rapports entre la condoléance et la consolation: "en somme, au dix-septième siècle les condoléances existent en tant que genre autonome dans le compliment de condoléance et en tant qu'exorde dans les lettres de consolation. En revanche, au dix-huitième siècle la lettre de consolation s'efface devant celle de condoléance qui inclura peut-être quelques topoï consolatoires ", op. cit., p. 222.

5. Nous renvoyons à l'enquête de R. Baustert, La Consolation érudite. Huit études sur les sources des lettres de consolation de 1600 à 1650, Tübingen, Gunter Narr, « Biblio 17 », 141, 2003, p. 45-139.

6. Parmi les marqueurs linguistiques caractéristiques de la consolation, N. Doiron signale notamment les adverbes et conjonctions de temps, l'impératif et la prolepse, «Poétique de la consolation classique. L'exemple du Recueil (1627) de Faret », XVII siècle, oct.-déc. 2007, nº 237, p. 779 et 789 .

7. D. Carabin distingue cinq sortes d'argumentaires qui «constituent le substrat cadre de la Lettre de consolation » : religieux, intellectuel, moral, psychologique et social, « Les Lettres de Nicolas Pasquier : la lettre de consolation », Revue d'Histoire Littéraire de la France (RHLF), janv.févr. 2002, 1, p. 24-25.

8. Pour T. Carr, « les lettres de consolation et les compliments de condoléance ont en commun un certain nombre de composantes: (1) expressions de la part qu'on prend à l'affliction de l'endeuillé ; (2) réflexions et exhortations consolatoires, soit morales, soit religieuses ; (3) louanges du défunt; et (4) offres de services ", "Se condouloir ou consoler ? Les condoléances dans les manuels épistolaires de l'ancien régime », op. cit., p. 220-221.

9. Ch. Noille, «La rhétorique de l'oraison funèbre : présentation, traduction, exemples », Papers on French Seventeenth Century Literature, 2015, vol. 82, p. 44.

10. Après avoir signalé «les recommandations de simplicité qui se multiplient vers la fin du siècle " (p. 225-226), Th. Carr synthétise l'évolution des tendances stylistiques de la lettre de consolation au cours $\mathrm{du} \mathrm{XVII}{ }^{\mathrm{e}}$ siècle en soulignant que "la lettre éloquente d'inspiration humaniste, où les arguments de consolation se déploient copieusement, cède le terrain aux condoléances qui peuvent satisfaire à la fois les obligations de l'honnêteté et le désir d'une expression plus personnelle ", «Se condouloir ou consoler ? Les condoléances dans les manuels épistolaires de l'ancien régime ", op. cit., p. 233.

11. S. Macé, "L'amplificatio, l'attenuatio et l'exercice de la conciliation: l'exemple des lettres et poèmes de conciliation du premier XVII ${ }^{\mathrm{e}}$ siècle ", dans S. Cornic et P. Servet dir., L'art de la conciliation, Genève, Droz, (« Cahiers de GADGES »), 2013, p. 129. Id., ibid., p. 128 : « [...] un discours de consolation typique comporte deux parties : la première consiste en quelque sorte à "se faire 
accepter" par la personne affligée ; ce n'est que dans un second temps que l'on peut s'autoriser à tenir un discours - moral, religieux, personnel - qui envisage les moyens de remédier au chagrin. »

12. Tantôt cette structuration bipartite est signalée par des connecteurs adversatifs, tels que néanmoins, mais toutefois ou mais pourtant, comme dans les manuels de Jean Puget de la Serre (Le Secrétaire à la Mode..., Amsterdam, Louis Elzevier, 1646, "Instruction à écrire des lettres ", «Lettres de consolation », p. 30-31) et de Pierre Richelet (Les plus belles lettres françoises sur toutes sortes de sujets, Tirés des meilleurs Auteurs, avec des Notes, tome II, Paris, Michel Brunet, 1698, «Réflexions sur la manière de consoler », p. 574). Tantôt ce principe de binarité est explicité par des connecteurs temporels, tels que d'abord ou après, comme c'est le cas pour les descriptifs de Paul Jacob (Le Parfait secrétaire..., Paris, Antoine de Sommaville, 1646, chap. IV, « Des préceptes de la Lettres de Consolation, ou Condoléance ", p. 87) et de Paul Colomiès (La Rhétorique de l'honnête homme..., Amsterdam, George Gallet, 1699, chap. IV, «La manière de faire une lettre de consolation", p. 23 et p. 24). Tantôt le double mouvement argumentatif propre au discours consolatoire prend la forme d'une concession, au moyen d'une relative à structure attributive de sens concessif, du type quelque... que, comme dans le patron rhétorique que propose René Milleran dans son "Instruction familière pour faire des lettres sur toutes sortes de sujets", Lettres familières, galantes, Et autres sur toutes sortes de sujets, avec leurs Réponses..., $2^{\text {nde }}$ édition, Bruxelles, Jean Leonard, 1692, XVI « De consolation », p. 386.

13. S. Macé, «L'amplificatio, l'attenuatio et l'exercice de la conciliation : l'exemple des lettres et poèmes de conciliation du premier XVII ${ }^{\mathrm{e}}$ siècle », op. cit., p. 130.

14. Comme le souligne D. Carabin, «la concession préférée à l'opposition forte permet de nuancer, d'autoriser le regret du passé tout en montrant que les lois du présent s'imposent. [...] L'usage de la concession fait songer à l'excuse, aux circonstances atténuantes que l'adulte ou un juge peuvent trouver à un mineur ou à un coupable affaibli ", « Les Lettres de Nicolas Pasquier : la lettre de consolation ", op. cit., p. 25-26.

15. R. Duchêne rappelle en note que c'est bien le troisième garçon que perd Mme de Grignan, après «l'enfant de la fausse couche de Livry en 1669 » et «celui qui mourut pendant l'accouchement en 1673, le prématuré de 1676 », note 1 de la page 481 (II, 1326).

16. Le risque d'aviver la douleur plutôt que de l'atténuer par de trop longs témoignages de condoléance constitue un thème récurrent dans les exemples de lettres de consolation fournis par les manuels. Citons le début de l'« Autre Billet de consolation d'une mère à son fils, sur la mort de sa femme » : «Si c'est une consolation aux personnes affligées de savoir qu'on prend part à leur douleur, assurez-vous que je suis sensible à la vôtre autant et plus que vous-même. Mais je crains bien que cette pensée, au lieu de diminuer votre affliction, ne l'augmente. Ainsi je ne vous en dis pas davantage [...] », Essais de Lettres familières Sur toute sorte de Sujets. Avec Un Discours sur l'Art Épistolaire. Et quelques remarques nouvelles sur la Langue Françoise, Paris, Jacques Le Febvre, 1690, dans A. Furetière, Nouvelle allégorique ou Histoire des derniers troubles arrivés au Royaume d'Éloquence (1659) - Essais de Lettres familières (1690), éd. M. Bombart et N. Schapira, Toulouse, Société de Littératures classiques, coll. « Rééditions de textes du XVII ${ }^{\mathrm{e}}$ siècle », supplément de la revue Littératures classiques, 2004, p. 65.

17. Sur la pratique du compliment en général et du compliment de condoléance en particulier, voir Th. Carr, «Se condouloir ou consoler ? Les condoléances dans les manuels épistolaires de l'ancien régime », op. cit., p. 218-219.

18. Le fait que le discours consolatoire n'échappe pas au dialogue épistolaire, y compris dans le cadre d'échanges plus formels qu'une correspondance familière entre une mère et sa fille, est confirmé par le soin que prennent plusieurs manuels d'art épistolaire à enseigner la manière dont il faut répondre à une lettre de consolation. Tantôt les manuels donnent des exemples de réponse, comme le fait Le Secrétaire à la mode de Puget de la Serre : la «Lettre de consolation à un père sur la mort de son fils » est suivie de deux réponses; la «Lettre de consolation à un fils sur 
la mort de son père » est suivie de quatre réponses; les trois «Lettres de consolation à un mari sur la mort de sa femme » sont suivies de cinq réponses; les trois «Lettres de consolation à une femme sur la mort de son mari» sont suivies de deux réponses, op.cit., p. 85-111. Tantôt les manuels donnent des consignes pour répondre à une lettre de consolation, à l'instar de Paul Jacob (qui fait suivre le chapitre IV intitulé «Des préceptes de la Lettre de Consolation, ou Condoléance » et l'exemple de lettre de consolation d'un chapitre V intitulé « Des Préceptes pour la Réponse" et d'un exemple de réponse, Le Parfait secrétaire, op.cit., p. 108-114) et de René Milleran (qui consacre un développement spécifique à la "Réponse aux Lettres de consolation » dans son «Instruction familière pour faire des lettres sur toutes sortes de sujets ", Lettres familières, galantes, op. cit., p. 393).

19. L'accusation qui consiste à présenter le chagrin persistant comme une forme de révolte contre la volonté divine constitue un lieu commun des lettres de consolation. Citons par exemple les remontrances et les menaces de l'«Autre Lettre de Consolation d'une veuve à une autre veuve, sur la mort de son fils aîné " procurée par Furetière : "Voulez-vous par vos pleurs vous opposer à la volonté de Dieu? Ce serait moyen d'attirer sa colère sur les enfants qui vous restent. ", Essais de Lettres familières, op. cit., p. 63.

20. J. Duchêne, " "Mes petites entrailles", ou l'art d'être grand-mère de Mme de Sévigné », op. cit., p. 56.

21. C'est à Jacques Du Roure que nous empruntons les formules de nos sous-titres, qui synthétisent les différentes matrices argumentatives mises en œuvre par Mme de Sévigné, La Rhétorique française, Paris, Chez l'auteur, 1662, p. 76.

22. «Il faut encore considérer le mal qu'elle [la personne qui est consolée] a reçu, et voir si elle l'a reçu nouvellement, ou depuis quelque temps, s'il est ou s'il n'est pas extrêmement sensible, s'il doit ou ne doit pas toujours durer. », id.

23. Nous renvoyons notamment à Jean Puget de la Serre (qui recommande de dire au destinataire endeuillé "Qu'il est Chrétien et doit se soumettre sans murmure à la volonté de Dieu ", Le Secrétaire à la Mode, op. cit., p. 31) ; à Paul Jacob (qui conseille de rappeler « que quand il plaît à la Providence de les ôter à qui que ce soit, il ne faut pas avoir le moindre soupçon d'injustice ", Le Parfait Secrétaire, op. cit., p. 88 ; à René Milleran («Vous tâcherez donc de le consoler [...] par les principales [considérations] qui se tirent ordinairement du Ciel, ou au pis aller, du temps qui est le plus souvent le meilleur remède à ces sortes de maux, et à la considération qu'on ne doit point murmurer contre la Providence divine", Lettres familières, galantes, op.cit., p. 384) ; à Paul Colomiès ( «Après, pour le rendre plus capable de consolation, il lui doit représenter que cette perte est un coup de la Providence divine, à laquelle il se doit soumettre ", La Rhétorique de l'honnête homme, op. cit., p. 23).

24. Les deux lettres de consolation adressées à un père et à une mère que donne en exemple Pierre Ortigue de Vaumorière illustrent la prédominance absolue des arguments religieux dans les lettres destinées à des parents endeuillés, Lettres sur toutes sortes de sujets, avec des avis sur la manière de les écrire, tome I, Paris, Jean Guignard, 1690, p. 94-100. La première («Lettre à un homme de qualité, sur la mort de son fils", op. cit., p. 96-97) consacre un long développement à la volonté de Dieu et à ses mystères. La seconde («Lettre à une Dame de qualité sur la mort de sa fille », op. cit., p. 99-100) ne repose que sur un seul argument, celui de la nécessité de faire de cette perte une offrande à Dieu. La seconde lettre est précédée de ce commentaire: «Je voudrais insérer des sentiments de piété dans ce que j'écrirais sur cette matière, principalement si je m'adressais à des personnes d'un sexe qui est appelé dévot par l'Église ; c'est pourquoi j'ai choisi l'exemple qui suit dans un excellent Auteur pour le rapporter ».

25. Le thème de la félicité des âmes après la mort fait partie des arguments consolatoires courants. Ainsi Paul Jacob recommande-t-il de dire «en second lieu que la mort est un port assuré, qui rend l'homme à sa vraie patrie, où il a toutes sortes de contentement, en partage ; que les biens et la vie même qu'il possédait ne lui étaient que prêtés [...]; Que le change est bien 
agréable et avantageux d'un point de vie à une Éternité bienheureuse : que c'est chose non seulement inutile, mais honteuse à un Chrétien, de vouloir comme rappeler en vie celui qui a fait une belle fin, pour lui faire sentir les aigreurs d'ici-bas, après les incomparables délices du Ciel ; ou si l'on n'a pas cette pensée, c'est faire tort à sa mémoire de pleurer sans relâche, comme si on croyait que sa condition fût plus malheureuse en l'autre vie [...]», Le Parfait Secrétaire, op. cit., p. 88-89. Sur le thème du bonheur de l'âme auprès des créatures célestes, voir R. Baustert, «L'audelà dans les lettres de consolation ", dans La Consolation érudite, op. cit., p. 222-241.

26. Le canevas rhétorique que propose René Milleran «sur la perte d'autres enfants que d'uniques, et d'autres parents, etc.» se caractérise par l'hypertrophie du thème de la félicité céleste dont jouit l'âme défunte : «Vous représenterez de plus, que quand les enfants naissent, nous ne savons pas quel sort ils auront, que si nous le savions, nous les étoufferions souvent pour leur éviter les désastres dont ils sont menacés, que ceux qui meurent de bonne heure sont bienheureux, puisqu'ils meurent au Seigneur, et sont délivrés des peines de ce monde [...]. Enfin, vous représenterez, que si l'on s'attriste de cette vie mortelle, on se doit réjouir en même temps de l'immortelle, que Dieu a partagée avec le défunt; la mort n'étant pas proprement la fin de la vie, mais bien le commencement d'une meilleure ; que Dieu n'a eu qu'un fils qu'il n'a pas épargné, tout Dieu qu'il fût aussi ; et que le défunt a franchi le pas si constamment, et si chrétiennement que sa mort a répondu à sa vie, que nous devons tous souhaiter par conséquent de passer les dernier moment aussi doucement, et aussi tranquillement que lui, qu'il est sorti de cette vallée de misères, et que nous y restons ; d'où on peut dire avec Saint Bernard, qu'il faut laisser pleurer les morts à ceux qui ne croient pas la Résurrection, et avec Drexellius, qu'on ne doit point répandre de larmes pour ceux qui sont portés par les Anges dans le Ciel [...] », Lettres familières, galantes, op. cit., p. 387-389. Dans la pratique, le thème du bonheur du défunt, généralement traité de manière lapidaire, prend volontiers la forme de comparaisons, comme dans l'« Autre Lettre de Consolation d'une veuve à une autre veuve, sur la mort de son fils aîné »: "Mettez fin à vos regrets, celui que vous pleurez est plus heureux sans doute que ses frères: il est mort chrétiennement, et cela vous doit être d'une grande consolation. ", Essais de Lettres familières, op. cit., p. 63.

27. Sur le thème des défunts qui «jouissent de la présence des anges» et qui «sont devenus anges eux-mêmes ", voir R. Baustert, "L'au-delà dans les lettres de consolation ", dans La Consolation érudite, op. cit., p. 231-233.

28. É. Gérard-Gailly, Les sept couches de Mme de Grignan, Paris, Éditions Albert, 1936, p. 35.

29. C'est ce dont témoigne l'« Autre Lettre de Consolation à une mère sur la mort de son fils, qui n'était âgé que de six mois » donnée en exemple par Antoine Furetière : "Vous ne cessez de pleurer et de gémir sans considérer que je vous croyais consolée. Votre douleur fait de la peine à M... qui a autrefois autant perdu que vous dans cette mort. Je suis extrêmement surpris, Madame, que vous ne reveniez pas de votre affliction; car bien que vous soyez mère, vous êtes une mère Chrétienne, et on a toujours remarqué en vous une solide piété. Le salut de ceux qui meurent dans un autre âge n'est pas certain : mais nous ne saurions douter du salut de cet enfant, que Dieu dans sa bonté vient de retirer du monde, et qu'il a voulu reprendre six mois après vous l'avoir donné : Si vous l'aimiez, Madame, vous souhaitiez son bonheur; et si vous souhaitiez son bonheur, comment n'êtes-vous pas bien aise qu'il soit heureux? Il est vraiment heureux en toutes manières, heureux de voir Dieu sans l'avoir offensé ; heureux de jouir de la victoire avant que d'avoir combattu ; heureux que le temps de son exil ait été si court, et ne l'ait pas engagé à tant de peines, où cette malheureuse vie nous expose tous les jours. Ha! Madame, pourquoi vous plaignez-vous, bien loin de vous en plaindre; et songez qu'il vaut mieux être un Ange dans le Ciel, que d'être un Prince sur la terre. ", Essais de Lettres familières, op. cit., p. 72-73.

30. «On emploie toutes les raisons imaginables pour divertir sa tristesse. Il y en a qui se contentent de faire voir que la mort n'est pas un mal : d'autres que ce n'est pas un grand mal ", P. Jacob, Le Parfait Secrétaire, op. cit., p. 90. 
31. «On peut encore considérer la personne que regrette celle que l'on console: Car c'est ordinairement dans la perte des parents et des amis, que cette sorte de discours a lieu. Il est mort jeune, mais il est mort heureux. Ses amis ne l'ont guère possédé, mais sa mort est la seule douleur qu'il leur ait jamais donnée. Ce que la vie a de fâcheux était en ce reste d'année, qu'il n'a point vues. », Du Roure, La Rhétorique française, op. cit., p. 77.

32. Th. Carr, «Se condouloir ou consoler? Les condoléances dans les manuels épistolaires de l'ancien régime », op. cit., p. 228.

33. Partant du constat que les lettres de consolation « servent à adoucir les maux et adversités de nos amis" mais que celles-ci "étant diverses", elles "ne peuvent être guéries d'un même remède ", Puget de la Serre propose deux canevas argumentatifs différents : l'un pour les cas où « le mal n'est pas grand », l'autre pour les cas où « quelque grand mal est arrivé à votre ami », Le Secrétaire à la Mode, op. cit., p. 29-30.

34. Les lettres de consolation données à titre d'exemples par Ortigue de Vaumorière apparaissent soigneusement classées. Viennent d'abord des lettres écrites à l'occasion de deuils plus ou moins sensibles ("sur la mort de son fils ", "sur la mort de sa fille », "sur la mort de son mari ", puis trois lettres sur la mort d'un "frère", entrecoupées par deux lettres «à un prisonnier de guerre»). Viennent ensuite des lettres écrites lors de différentes épreuves (trois lettres "à un ami malade » et une "à un Ami dont on avait critiqué les ouvrages ») puis des lettres concernant des deuils moins douloureux (« sur la mort d'une de ses Amies », «À une Dame qui avait perdu sa Mère ", " À un Grand Seigneur, qui avait perdu son Père »). Après la «Lettre d'un fils qui avait perdu son père d'un âge très avancé ", la série d'exemples se termine par des lettres de consolation qui font la part belle au badinage et à la raillerie (telle que la «Plaisanterie sur une consolation »), dont Ortigue de Vaumorière commente et critique le style galant, Lettres sur toutes sortes de sujets, op. cit., p. 94-131.

35. R. Milleran, Lettres familières, galantes, op. cit., p. 385. Après avoir fait cette distinction, Milleran donne des préceptes pour écrire des lettres de consolation «Sur la maladie d'un ami » (XVII), "Sur la perte d'autres enfants que d'uniques, et d'autres parents, etc.» (XVIII), "Sur la perte d'une personne décrépite et cassée de vieillesse, ou d'une longue et fâcheuse maladie, et sans aucune espérance de retour » (XIX), "Sur la perte des biens » $(\mathrm{XX})$, « Sur la perte d'un procès » (XXI), op.cit., p. 386-393. Une fois encore, les occasions d'écrire des lettres de consolation sont classées en fonction de la gravité de la perte subie par le destinataire.

36. C'est dans une lettre adressée à Bussy-Rabutin que Mme de Grignan fait cette réflexion, au milieu de plaisanteries sur son accouchement prématuré : "On est bien moins de temps à recevoir des réponses de Québec que vous ne serez à recevoir celle-ci ; mais je serai entièrement justifiée auprès de vous si vous voulez bien ajouter, à tout le chemin qu'elle va faire, l'incident d'un accouchement, qui s'est placé mal à propos entre votre lettre et celle-ci. En lisant la supputation que vous me faisiez sur les couches de madame votre fille, il me prit une si violente envie d'accoucher que toute la supputation que je faisais de n'être qu'à huit mois ne fut pas capable de m'en empêcher. Si j'avais su que vos lettres eussent la même vertu que les reliques de sainte Marguerite, je vous aurais prié de différer d'un mois la joie que j'ai eue d'en recevoir. Mais après avoir fait l'expérience du bonheur que j'ai eu d'être heureusement délivrée d'un fils, qui vit contre toutes les règles de la médecine, vous pouvez m'écrire en tout temps [...] 》 (15 mars 1676, II : 268). Ce ton badin était déjà celui de Bussy-Rabutin se réjouissant avec Mme de Sévigné de l'heureux dénouement de l'accouchement prématuré de sa nièce : «Je suis fort aise que la belle Madelonne se porte bien de son accouchement à huit mois, et que son enfant vive. Comme elle s'est tirée du pair d'avec les autres femmes par son mérite, elle s'en veut tirer par toutes ses actions. » (15 avril $1676:$ II, 268-269).

37. Dans les lettres qui suivent la naissance du petit prématuré, Mme de Sévigné oscille entre le soulagement («[...] et je vois par là votre très bonne santé (c'était le dixième de votre couche), et en même temps celle de votre petit. ", $1^{\text {er }}$ mars 1676 : II, 245); le fatalisme sans espoir 
( J'embrasse de tout mon cœur Mme de de Coligny ; je la prie de ne pas accoucher à huit mois, comme ma fille. Elle s'en porte bien, mais on y perd un fils, et c'est dommage. », à Bussy-Rabutin, $1^{\mathrm{er}}$ mars 1676, II, 246) ; la perplexité ( «Je suis ravie de la santé du petit garçon, mais je n'ose m’y attacher, parce que je n'ose espérer que vous vous soyez trompée ; vous êtes plus infaillible que le pape. Je fonde donc toute mon espérance sur les contes à dormir debout que l'on vous fait à Aix ; je les trouve extrêmement plaisants, et la rareté des enfants de neuf mois m'a fait rire. " ", 4 mars 1676, II : 247) ; l'espérance («Je prends goût à la vie du petit garçon; je voudrais bien qu'il ne mourût pas. ", 8 mars 1676, II : 249) ; l'étonnement ("Je suis plus étonnée qu'une autre de la santé du petit enfant, car je me fie fort à vos supputations, et je trouve vos réponses fort plaisantes. Mais enfin ce sera donc un miracle si nous conservons cet enfant. » 15 mars 1676: II, 253); la confiance ( Je suis ravie que le petit enfant se porte bien; Villebrune dit qu'il vivra fort bien à huit mois, c'est-à-dire huit lunes passées. », 22 mars 1676 : II, 257) ; « [...] je vous avertis que, sans miracle, le petit d'Adhémar vivra fort bien cent ans. Vous me marquez le 15 juin. Nous avons supputé les lunes jusqu'au 9 février ; il est de deux jours dans la neuvième, c'est assez. ", 28 mars 1676 : II, 259) ; l'optimisme («Le petit petit, c'est-à-dire le gros gros, est un homme admirable ; je l'aime trop d'avoir voulu vivre contre vent et marée. », 15 avril 1676 : II, 1676) ; l'incrédulité (« Et ce petit, veut-il vivre absolument contre l'avis d'Hippocrate et de Galien ? Il me semble que ce doit être un homme tout extraordinaire. L'inhumanité que vous donnez à vos enfants est la plus commode chose du monde [...] », 4 juin 1677 : II, 312).

38. E. Méron, «Les enfants aux yeux des adultes, selon les lettres de Mme de Sévigné », dans R. Duchêne dir., Mme de Sévigné (1626-1696). Provence, spectacles, «lanternes ». Colloque international du Tricentenaire de la mort de Mme de Sévigné, Château de Grignan (29 mai-1 ${ }^{\text {er }}$ juin 1996), Grignan, Association d'Action Culturelle des Châteaux Départementaux de la Drôme, 1998, p. 306.

39. J. Duchêne, «"Mes petites entrailles”, ou l'art d'être grand-mère de Mme de Sévigné », op. cit., p. 60.

40. Nous employons ce terme en référence aux catégories rhétoriques utilisées par Érasme. C'est après les consignes théoriques sur le discours consolatoire, un florilège d'exemples tirés de Cicéron et de Pline le Jeune, puis des silves de son cru, qu'Érasme propose un exemple forgé de "Consolation à un proche avec objurgation", "Consolatio familiaris cum objurgatione mea " (trad.Ph. Collé et Ch. Noille: voir dans le présent numéro d'Exercices de Rhétorique, section ATELIER). Pour Jean-Claude Margolin, il s'agit d'une « variante de la consolation familière, celle qu'accompagnent des reproches amicaux et bourrus, destinés à "secouer" l'ami affligé », De conscribendis epistolis, éd. J.-Cl. Margolin, dans Opera Omnia Desiderii Erasmi Roterodami, Ordinis Primis, Tomus Secundus, Amsterdam, North-Holland Publishing Company, 1971, p. 462 (LB I 433), note 3.

41. L'emploi de la raillerie fait partie des ressources rhétoriques que préconise Puget de la Serre (Le Secrétaire à la Mode, op. cit., p. 29-30) lorsque «le mal n'est pas grand»: «Les Lettres de consolation servent à adoucir les maux et adversités de nos amis : qui étant diverses ne peuvent être guéries d'un même remède. En général, si le mal n'est pas grand, il leur faut dire qu'ils n'ont point sujet de se tant attrister, que la chose ne le mérite point : qu'ils doivent montrer plus de courage ; qu'ils font tort à leur réputation. Et même on peut entremêler quelque raillerie parmi son discours, pourvu qu'on sache que celui à qui elle s'adresse ne s'en offensera point. »

42. Citons l'exemple de «Lettre de Consolation d'un père à sa fille, sur la mort de son mari » procuré par Furetière, qui commence et se termine par d'âpres remontrances (Essais de Lettres familières, op.cit., p. 69-70) : «Je ne saurais condamner sans injustice les afflictions légitimes; mais les plus justes, comme est la vôtre, peuvent avoir leur excès, et c'est cet excès que je condamne. On nous écrit que nous ne voulez écouter ni conseil, ni raison, et que vous vous abandonnez au désespoir. [...] Je n'ai plus rien à vous dire en l'état où l'on me mande que vous êtes, et si malgré vos faiblesses je ne pouvais empêcher que mon cœur ne fût toujours le même à 
votre égard, je ne pourrais non plus empêcher qu'il ne fût pénétré d'une vive douleur de votre opiniâtreté. »

43. Voir Érasme, De conscribendis epistolis, op. cit., p. 435 (LB I 427, trad. Ph. Collé et Ch. Noille dans l'Atelier de ce numéro): "Dans la consolation, la plaisanterie sera absolument à proscrire, à moins qu'il ne s'agisse d'une légère infortune, et dans ce cas on pourra le dérider avec une plaisanterie. Si nous écrivons ainsi, pourvu que celui à qui nous écrivons soit du genre à être charmé par de telles choses, il faudra systématiquement plaisanter en caressant de sorte que nous mêlerons néanmoins de véritables consolations aux plaisanteries. » (In consolando jocus erit vehementer fugiendus, nisi leve sit incommodum, ut joco possit exhilarari. Tum si modo cui scribimus, is erit, qui hujusmodi rebus capiatur, quam blandissime jocandum erit, ita tamen, ut veras consolationes cum jocis permisceamus.)

44. Du Roure, La Rhétorique française, op. cit., p. 77: «Enfin on peut considérer les autres personnes qui touchent celle dont on veut diminuer la douleur. Ne désobligez pas messieurs vos autres frères avec des plaintes, qui leur fassent croire que vous préférez ce que vous avez perdu, à ce qui vous est demeuré. »

45. Ménager son père en mettant des bornes à son chagrin de veuve : tel est l'argument central de la « Lettre de Consolation d'un père à sa fille, sur la mort de son mari » proposée par Furetière (Essais, op. cit., p. 69-70) : «Prétendez-vous par cette conduite avancer mes jours et les vôtres? Songez du moins à ménager un père qui ne saurait voir sans s'étonner que vous fassiez paraître des sentiments tout contraires à ceux de votre époux, et que la femme d'un homme qui est mort si généreusement, après avoir témoigné tant de courage durant sa vie, témoigne dans son malheur si peu de force et de générosité.»

46. Citons l'« Autre Lettre de Consolation à Madame*** » du manuel de Furetière, qui insiste sur la nécessité de surmonter l'affliction du veuvage pour être en mesure de prendre soin de ses enfants (ibid., p. 70-71): «Si vous aimiez Monsieur [...] vous devez aimer ses enfants, et considérer qu'il vous est impossible de vous appliquer au soin de leur éducation, à moins que de modérer la douleur qui vous accable. Ainsi, Madame, faites effort sur vous-même ; servez-vous des lumières d'un esprit qu'on a toujours trouvé si raisonnable, et après la perte que votre famille vient de faire, et qui n'est que trop grande, ne lui en faites pas appréhender une seconde?»

47. Dans sa «Lettre à Monsieur de Termes sur la mort de son fils » (lettre citée par Puget de la Serre dans Le Secrétaire à la Mode, op. cit., p. 182-183), Malherbe met en garde son destinataire contre la «diminution de son honneur » qui résulterait d'une affliction persistante, avant de détailler les membres de son entourage que le destinataire endeuillé doit songer à protéger : d'abord ses enfants (" ayez-en le même soin et vivez pour leur donner le même secours ») ; puis son épouse (« et vous en conjure encore par l'affection extrême que vous avez toujours portée à Madame votre femme. Vous lui devez toute sorte de bons exemples; donnez-lui celui de se conformer à la volonté de Dieu : Et craignez que vous voyant si opiniâtre à vous affliger, elle qui est d'un sexe où il semble que la tendresse de cœur soit une louange, ne se porte à des extrémités qui ajoutent un second malheur à celui qui vous est arrivé »); et enfin son frère (« Finalement, Monsieur, souvenez-vous que vous avez un frère, que non seulement notre Cour, mais toutes les Cours étrangères prennent pour un Patron de vertu. Vous lui avez des obligations aussi grandes, que vous les sauriez désirer d'un Père: Portez-lui ce respect de croire que quoique la fortune vous ôte, vous aurez toujours assez tant qu'elle vous le conservera »). Ce passage est repris et légèrement modifié par Ortigue de Vaumorière, qui, après avoir à son tour évoqué les enfants et l'épouse, attire l'attention sur le père et sur le monarque que le destinataire endeuillé doit prendre soin de ménager (Lettres sur toutes sortes de sujets, op. cit., p. 97-98) : « Souvenez-vous aussi que vous avez un père estimé de toute la Cour, et dont vous recevez tous les jours des bienfaits. Témoignez-lui que malgré tout ce que la fortune vous ôte, vous aurez toujours assez, tant qu'elle vous le conservera. Ajoutez à ces considérations celles qui regardent le Grand Monarque que vous 
servez. Il vous confie des emplois considérables dans les Armées, et cet honneur qu'il vous fait vous oblige à ne connaître d'autre intérêt que le sien. »

48. Sur les supplications auxquelles donnent lieu les inquiétudes de Mme de Sévigné au sujet de la santé fragile de sa fille, voir C. Lignereux, «Les supplications maternelles à l'épreuve des convenances épistolaires », dans L. Albert, P. Bruley et A.-S. Dufief dir., La supplication. Discours et représentation, Rennes, PUR, coll. «Interférences », 2014, p. 29-39.

49. Du Roure, La Rhétorique française, op. cit., p. 77 : «Enfin on tâche à diminuer le regret des choses passées, par l'espérance des Futures. »

50. Pour Grimarest, qui recommande « que l'on n'exagère point une perte " (Traité sur la manière d'écrire des lettres, et sur le cérémonial: Avec un Discours sur ce qu'on appelle Usage dans la Langue Françoise [1709], Paris, Veuve Estienne, 1735, p. 68), «c'est même une adresse obligeante de ne point se servir en cette occasion de termes qui représentent trop vivement la perte que l'on a faite, pour ne point rappeler ces moments rigoureux d'affliction, qui sont toujours aisés à faire renaître dans le cœur des personnes qui sont sincèrement touchées. "

51. Nous reprenons la formule de Claude Irson, qui synthétise les analyses d'Érasme, dans sa "Methode pour bien ecrire \& composer des lettres, que l'on appelle epistres ", contenue dans la Nouvelle Methode pour apprendre facilement les principes et la pureté de la langue françoise, Contenant plusieurs traitez De la prononciation, De l'Orthographe, De l'Art d'Ecriture, Des Etymologies, Du Stile Epistolaire, \& Des Regles de la belle façon de Parler \& d'Ecrire [Paris, Gaspard Meturas, 1656], Genève, Slatkine, 1973, p. 242. Le passage d'Irson est repris par François de Fenne dans Le Secrétaire à la mode réformé ou le Mercure nouveau Contenant les lettres Choisies des plus beaux Esprits de ce Tems. Avec une nouvelle Instruction à écrire des lettres, \& VI Entretiens de la Civilité, Leyde, Jacques Hackius, 1684, p. 26.

52. Selon Paul Colomiès (La Rhétorique de l'honnête homme, op. cit., p. 23), « celui qui veut faire une lettre pour consoler quelqu'un [...] doit parler des autres biens que la Providence lui a laissés, pour se dédommager de ceux qu'il vient de perdre. »

53. René Milleran (Lettres familières, galantes, op.cit., p. 387) recommande de dire «que, comme [Dieu] ne nous a mis au monde que pour mourir, et après cette mort, vivre éternellement, il était en droit, nous ayant donné nos enfants, de nous les ôter, qu'il nous en redonne d'autres quand il lui plaît, qui nous sont souvent plus agréables que ceux-là, que des pierres, Dieu peut susciter des enfants à Abraham. »

54. Dans la lettre intitulée «Autre Lettre de Consolation d'une veuve à une autre veuve, sur la mort de son fils aîné » (Furetière, Essais de Lettres familières, op. cit., p. 63), l'épistolière se sert de cet argument pour morigéner une destinataire dont elle juge le chagrin excessif : « [...] et pardessus cela vous avez des enfants de grande espérance, et moi je n'ai rien de tout cela : beaucoup de dettes à payer, et point d'enfants pour partager les peines et les ennuis de mon veuvage. »

55. Après la lettre de consolation "d'un Père à un de ses amis sur la mort de son propre Fils qui lui avait donné beaucoup de chagrin durant sa vie », Furetière donne la réponse à cette lettre (Essais de Lettres familières, op. cit., p. 75), qui se clôt ainsi : «Je vous plaindrais s'il ne vous en restait plus: mais vous en avez d'autres qui sont plus sages, et qui vous donnent, et vous donneront toujours de la satisfaction. "

56. Voir Érasme, De conscribendis epistolis, op. cit., p. 434 (LB I 427, trad. Ph. Collé et Ch. Noille dans l'ATELIER de ce numéro) : "Nous atténuerons le malheur si nous montrons qu'il sera de courte durée. Car nous supportons habituellement avec plus de patience les infortunes que nous pensons devoir être bientôt finies, surtout s'il est montré que cette infortune sera suivie de quelque grande espérance. À ce point nous examinerons avec le plus grand soin toutes les conjectures par lesquelles nous rendrons cette espérance au plus haut point probable. » (Malum extenuabimus, si minime diuturnum fore ostendemus. Solent enim patientius ferri incommoda, quae brevi finem habitura putentur, praesertim si eam incommoditatem magna aliqua spes sequutura monstretur. Hic 
omnes rerum conjecturas diligentissime dispiciemus, quibus eam spem quam maxime probabilem efficiamus.)

57. Dans sa «Lettre à Monsieur de Termes, sur la mort de son fils ", Malherbe écrit : « Vous avez satisfait à la mémoire du fils que vous avez perdu, pensez à ceux qui vous sont demeurés. Ils sont branches de la même souche, et vous donnent les mêmes espérances : ayez-en le même soin et vivez pour leur donner le même secours. » Cette lettre est citée aussi bien par Puget de la Serre (Le Secrétaire à la Mode, op. cit., p. 181-182) que par Ortigue de Vaumorière (Lettres sur toutes sortes de sujets, op. cit., p. 97).

58. Nous reprenons les formules de Puget de la Serre, Le Secrétaire à la Mode, op. cit., p. 30.

59. Paul Colomiès clôt l'argumentaire standard qu'il préconise de mettre en œuvre pour les lettres de consolation «les plus ordinaires» par la mise en garde suivante (La Rhétorique de l'honnête homme, op. cit., p. 24-25) : «On ne doit pas toujours se servir de toutes ces raisons. Il faut se servir des plus convenables ».

60. Le manuel d'art épistolaire de Jean Puget de la Serre, qui connut un succès considérable tout au long du siècle, comprend un Recueil de Lettres morales des plus beaux esprits de ce temps (Le Secrétaire à la Mode, op. cit., p. 143-266). Cette anthologie de vingt-six lettres commence par quinze lettres de consolation - les onze premières à propos de deuils, la douzième sur un emprisonnement, les deux suivantes sur des maladies et la dernière sur une disgrâce - écrites par les plus prestigieux hommes de lettres de l'époque baroque (citons notamment le cardinal du Perron, François de Rosset, Guillaume du Vair, François de Malherbe, Jean Auvray, François Le Métel de Boisrobert ou encore Antoine Godeau). Nous renvoyons en particulier aux trois lettres de consolation écrites à l'occasion de la mort d'enfants : «À Monsieur de Termes, sur la mort de son fils » de Malherbe (p. 178-184), «À un Magistrat, sur la perte de deux de ses enfants morts de peste » d'Auvray (p. 184-195) et «À un Père, sur la perte de tous ses enfants » du même Auvray (p. 195-202).

61. P. Jacob, Le Parfait Secrétaire, op. cit., p. 95.

62. Du vivant de Mme de Sévigné, les critiques dont font l'objet les lettres de consolation, jugées aussi artificielles qu'inutiles, ne manquent pas - qu'elles interviennent dans le cadre d'une correspondance mondaine, d'un ouvrage sur l'art de la conversation ou dans un manuel épistolographique. Dès 1644, Mlle de Scudéry se révolte contre un rituel qu'elle juge sclérosé en refusant de s'acquitter des lettres de consolation qu'elle devrait écrire à Mlles de Clermont et à leur mère : « [...] il y a si longtemps que la mort est introduite dans le monde et qu'il y des gens qui en écrivent et qui en parlent, que je ne trouve plus rien à en dire ", lettre à Angélique Paulet citée par D. Denis dans Madeleine de Scudéry, "De l'air galant " et autres Conversations. Pour une étude de l'archive galante, éd. D. Denis, Paris, Champion, «Sources classiques », 1998, p. 150, note 6. En 1677, dans son Discours de la conversation, le chevalier de Méré raille le caractère ridicule de phraséologies aussi prévisibles que dévaluées: «Il ne faut pourtant pas qu'elles soient si communes que celle-ci, que tout le monde sait par cœur ; la part que je prends à votre déplaisir ; j'ai vu parier en ouvrant une lettre de consolation, que cela s'y trouverait ; et une Dame fort triste qui l'avait reçue, ne pût s'empêcher d'en rire ", cité par S. Macé dans «L'amplificatio, l'attenuatio et l'exercice de la conciliation : l'exemple des lettres et poèmes de conciliation du premier XVII siècle ", op. cit., p.134-135, note 29. En 1690, c'est à l'intérieur d'une lettre dans laquelle l'épistolier évoque la mort de son père qu'Ortigue de Vaumorière glisse des sarcasmes à l'encontre de pratiques épistolaires discréditées par leur hypocrisie (Lettres sur toutes sortes de sujets, op. cit., p. 122-123, «Lettre d'un fils qui avait perdu son père d'un âge très avancé ») : «Je vous prie de ne point parler de la mort de mon père, une infinité de gens de cette Province que je ne connaissais point, ont pris cette occasion pour m'écrire, et je vois bien que leurs Lettres n'ont que des réponses pour but. Voilà qui est étrange! me vouloir écrire sans affaire et sans amitié : J'aimerais encore mieux qu'ils me vinssent voir, j'en serais quitte pour un repas ou deux. » 
63. À Amilcar qui demande à Plotine de lui enseigner l'art de faire des lettres de consolation, Plotine répond (Madeleine de Scudéry, "De la manière d'écrire des lettres», op. cit., p. 149-150) : «[...] je ne puis souffrir ces grandes lettres de consolation qui n'ont jamais nul effet : cependant on dirait à entendre parler certaines gens, qu'ils prétendent que leurs lettres aient une force magique contre l'affliction, et qu'en les lisant on doit ne sentir plus nul regret de ce qu'on a perdu. Je voudrais pourtant bien, ajouta-t-elle, qu'on se persuadât une fois pour toutes qu'il n'appartient qu'au temps de consoler de semblables douleurs, et que ce n'est point à l'éloquence à s'en mêler. »

64. R. Milleran, Lettres familières, galantes, op. cit., p. 383.

65. Pour Plotine (Madeleine de Scudéry, « De la manière d'écrire des lettres », op. cit., p. 150), « il ne faut simplement que témoigner à la personne à qui l'on écrit, la part qu'on prend à sa douleur, sans aller faire de longues plaintes [...] ». Quant à Amilcar, il « fai[t] vœu [...] de ne faire plus de longues exagérations contre la cruauté de la mort, comme font certaines gens [...]. »

66. P. Jacob, Le Parfait Secrétaire, op. cit., p. 93 : «Il faut prendre garde de ne ressembler pas à certains consolateurs si sérieux, et si importuns, qu'ils redoublent les afflictions, en voulant consoler : on évitera aussi le commandement. »

67. P. Ortigue de Vaumorière, Lettres sur toutes sortes de sujets, op. cit., p. 90 : «C'est dans ce genre d'écrire qu'il faut que le cœur paraisse touché, et qu'on l'excite à parler sans le secours de l'esprit. Montrons-nous dans ces occasions moins spirituels que sensibles. Choisissons des expressions tendres et naturelles, et rejetons les pensées qui peuvent avoir quelque chose de brillant ou de trop recherché. »

68. Pour Grimarest (Traité sur la manière d'écrire des lettres, op. cit., p. 69), les lettres de consolation «embarrassées [...] d'oraisons funèbres, sont d'un très mauvais goût, et révoltent les personnes de bon esprit. »

69. P. Colomiès, La Rhétorique de l'honnête homme, op. cit., p. 22 : «Les expressions naturelles et tendres sont les seules qu'on doit employer dans les lettres de consolation. Il faut que le cœur y parle seul ; qu'il n'y ait rien de trop brillant, ni qui paraisse trop recherché. »

70. Nous reprenons les formules de Plotine (Madeleine de Scudéry, «De la manière d'écrire des lettres», op.cit., p. 150): "C'est pourquoi je trouve que le mieux qu'on puisse faire en ces occasions, est de faire les lettres de consolation fort courtes : car pour en écrire de raisonnables, il ne faut simplement que témoigner à la personne à qui l'on écrit, la part qu'on prend à sa douleur, sans aller faire de longues plaintes, ou de grands éloges : et sans employer toute la Morale, et toute l'éloquence inutilement. »

\section{AUTEUR}

\section{CÉCILE LIGNEREUX}

Université Grenoble Alpes - UMR Litt\&Arts - RARE Rhétorique de l'Antiquité à la Révolution 